\title{
HYPERBOLIC DIFFERENTIAL-OPERATOR EQUATIONS ON A WHOLE AXIS
}

\author{
YAKOV YAKUBOV
}

Received 24 August 2003

We give an abstract interpretation of initial boundary value problems for hyperbolic equations such that a part of initial boundary value conditions contains also a differentiation on the time $t$ of the same order as equations. The case of stable solutions of abstract hyperbolic equations is treated. Then we show applications of obtained abstract results to hyperbolic differential equations which, in particular, may represent the longitudinal displacements of an inhomogeneous rod under the action of forces at the two ends which are proportional to the acceleration.

\section{Introduction}

The first attempt to give an abstract interpretation of hyperbolic problems such that a part of boundary value conditions may contain the differentiation on the time was done in [8] for almost periodic solutions and oscillations decay cases, and in [5] for hyperbolic differential-operator equations on a finite interval. In this paper, we continue this study to the case of differential-operator equations on a whole axis. In particular, we find sufficient conditions for which the solution of the considered problems is stable.

Let $H$ and $F$ be Hilbert spaces. The set $H \oplus F$ of all vectors of the form $(u, v)$ where $u \in H$ and $v \in F$, with usual coordinatewise linear operations and the norm

$$
\|(u, v)\|_{H \oplus F}:=\left(\|u\|_{H}^{2}+\|v\|_{F}^{2}\right)^{1 / 2}
$$

is a Hilbert space and called the orthogonal sum of Hilbert spaces $H$ and $F$.

For the operator $A$ closed in a Hilbert space $H$, the domain $D(A)$ is turned into a Hilbert space $H(A)$ with respect to the norm

$$
\|u\|_{H(A)}:=\left(\|u\|^{2}+\|A u\|^{2}\right)^{1 / 2} \text {. }
$$

If $H_{1}$ and $H$ are two Hilbert spaces where $H_{1} \subset H$, then $H_{1}$ can be represented as the domain $D(S)=H_{1}$ of a suitable positive definite selfadjoint operator $S$ in $H$ (see, e.g., 
[4, Remark 1.18.10/3]). Then, by [4, Theorem 1.18.10], the interpolation space

$$
\left(H_{1}, H\right)_{\theta, 2}=H\left(S^{1-\theta}\right)
$$

\section{Hyperbolic differential-operator equations}

We give, in this section, an abstract interpretation of initial boundary value problems for hyperbolic equations such that a part of boundary value conditions contains also the differentiation on the time $t$.

Let $H$ and $H^{\nu}, v=1, \ldots, s$, be Hilbert spaces. Consider the following Cauchy problem (abstract "initial boundary value problem"):

$$
\begin{gathered}
L\left(D_{t}\right) u:=u^{\prime \prime}(t)+B u(t)=0, \\
L_{v}\left(D_{t}\right) u:=\left(A_{\nu 0} u(t)\right)^{\prime \prime}+A_{\nu 2} u(t)=0, \quad v=1, \ldots, s, \\
u(0)=u_{0}, \quad u^{\prime}(0)=u_{1},
\end{gathered}
$$

where $t \in \mathbb{R} ; B$ is an operator in $H ; A_{\nu 0}$ and $A_{\nu_{2}}$ are operators from a subspace of $H$ into $H^{\nu}$; and $u(t)$ from $\mathbb{R}$ into $H$ is an unknown function. Note that operators $B, A_{\nu 0}$, and $A_{\nu 2}$ are, generally speaking, unbounded.

A function $u(t)$ is called a solution of problem (2.1) if the function $t \rightarrow\left(u(t), A_{10} u(t), \ldots\right.$, $\left.A_{s 0} u(t)\right)$ from $\mathbb{R}$ into $H \oplus H^{1} \oplus \cdots \oplus H^{s}$ is twice continuously differentiable, from $\mathbb{R}$ into $H(B) \oplus H^{1} \oplus \cdots \oplus H^{s}$ is continuous, and $u(t)$ satisfies (2.1).

We say that problem (2.1) is stable if each of its solution $u(t)$ with $u_{0} \in H(B), u_{1} \in$ $H(B)$, is bounded, that is,

$$
\|u(t)\| \leq C, \quad t \in \mathbb{R} .
$$

Consider a system of operator pencils corresponding to (2.1a) and (2.1b);

$$
\begin{gathered}
L(\lambda):=\lambda I+B, \\
L_{\nu}(\lambda):=\lambda A_{\nu 0}+A_{\nu 2}, \quad \nu=1, \ldots, s,
\end{gathered}
$$

where $\lambda$ is a complex number.

THEOREM 2.1. Let the following conditions be satisfied:

(1) $B$ is a closed operator in a Hilbert space $H$ with a dense domain $D(B)$; the embedding $H(B) \subset H$ is compact;

(2) the operators $A_{\nu 0}$ from $(H(B), H)_{1 / 2,2}$ into $H^{v}$ act boundedly and $A_{v 2}, v=1, \ldots, s$, from $H(B)$ into $H^{v}$ act boundedly;

(3) the linear manifold $\left\{v \mid v:=\left(u, A_{10} u, \ldots, A_{s 0} u\right), u \in D(B)\right\}$ is dense in the Hilbert space $H \oplus H^{1} \oplus \cdots \oplus H^{s}$; 
(4) for all $u \in D(B), v \in D(B)$,

$$
\begin{aligned}
& (B u, v)_{H}+\left(A_{12} u, A_{10} v\right)_{H^{1}}+\cdots+\left(A_{s 2} u, A_{s 0} v\right)_{H^{s}} \\
& =(u, B v)_{H}+\left(A_{10} u, A_{12} v\right)_{H^{1}}+\cdots+\left(A_{s 0} u, A_{s 2} v\right)_{H^{s}}
\end{aligned}
$$

(5) for all $u \in D(B)$,

$$
0 \leq(B u, u)_{H}+\left(A_{12} u, A_{10} u\right)_{H^{1}}+\cdots+\left(A_{s 2} u, A_{s 0} u\right)_{H^{s}} \leq C\|u\|_{(H(B), H)_{1 / 2,2}}^{2} ;
$$

(6) some real number $\lambda_{0}$ is a regular point for the operator pencil $\mathbb{L}(\lambda): u \rightarrow \mathbb{L}(\lambda) u:=$ $\left(L(\lambda) u, L_{1}(\lambda) u, \ldots, L_{s}(\lambda) u\right)$, which acts boundedly from $H(B)$ onto $H \oplus H^{1} \oplus \cdots \oplus H^{s}$;

(7) $\left(u_{1}, A_{10} u_{1}, \ldots, A_{s 0} u_{1}\right) \in \operatorname{Im}\left(\mathbb{B}^{1 / 2}\right)$, where

$$
\begin{gathered}
D(\mathbb{B}):=\left\{v \mid v:=\left(u, A_{10} u, \ldots, A_{s 0} u\right), u \in D(B)\right\} \\
\mathbb{B}\left(u, A_{10} u, \ldots, A_{s 0} u\right):=\left(B u, A_{12} u, \ldots, A_{s 2} u\right)
\end{gathered}
$$

is an operator in the Hilbert space $\mathcal{H}:=H \oplus H^{1} \oplus \cdots \oplus H^{s}$ and from condition (5), it follows that $(\mathbb{B} v, v) \geq 0$, for all $v \in D(\mathbb{B})$;

(8) $u_{0} \in H(B), u_{1} \in(H(B), H)_{1 / 2,2}$.

Then there exists a unique solution $u(t)$ of problem (2.1) such that the function $t \rightarrow$ $\left(u(t), A_{10} u(t), \ldots, A_{s 0} u(t)\right)$ from $\mathbb{R}$ into $H \oplus H^{1} \oplus \cdots \oplus H^{s}$ is twice continuously differentiable, and from $\mathbb{R}$ into $H(B) \oplus H^{1} \oplus \cdots \oplus H^{s}$ is continuous, and for $t \in \mathbb{R}$, the following estimate holds:

$$
\begin{gathered}
\|u(t)\|+\left\|u^{\prime \prime}(t)\right\|+\sum_{\nu=1}^{s}\left\|\left(A_{\nu 0} u(t)\right)^{\prime \prime}\right\|_{H^{v}}+\|B u(t)\| \\
\leq C\left(\left\|B u_{0}\right\|+\left\|u_{0}\right\|+\left\|u_{1}\right\|_{(H(B), H)_{1 / 2,2}}\right)
\end{gathered}
$$

consequently, problem (2.1) is stable.

Proof. Consider, in the Hilbert space $\mathscr{H}:=H \oplus H^{1} \oplus \cdots \oplus H^{s}$, the above-mentioned operator $\mathbb{B}$. Then the Cauchy problem

$$
\begin{gathered}
v^{\prime \prime}(t)+\mathbb{B} v(t)=0, \\
v(0)=v_{0}, \quad v^{\prime}(0)=v_{1},
\end{gathered}
$$

is equivalent to the Cauchy problem $(2.1)$, where $v_{0}:=\left(u_{0}, A_{10} u_{0}, \ldots, A_{s 0} u_{0}\right), v_{1}:=\left(u_{1}\right.$, $\left.A_{10} u_{1}, \ldots, A_{s 0} u_{1}\right)$. Indeed, let $u(t)$ be a solution of problem (2.1). Then, $v(t)=\left(u(t), A_{10} u(t)\right.$, $\left.\ldots, A_{s 0} u(t)\right)$ is a solution of problem (2.8). Conversely, let $v(t)$ be a solution of problem (2.8). Since $v(t) \in D(\mathbb{B})$, then $v(t):=\left(u(t), A_{10} u(t), \ldots, A_{s 0} u(t)\right)$, where $u(t) \in D(B)$, for all $t \in \mathbb{R}$. Substituting $v(t)$ into (2.8), we get that $u(t)$ satisfies (2.1). 
102 Hyperbolic differential-operator equations on a whole axis

By virtue of condition $(3), D(\mathbb{B})$ is dense in $\mathscr{H}$. By virtue of condition (4), for $\tilde{v}_{1}=$ $\left(\tilde{u}_{1}, A_{10} \tilde{u}_{1}, \ldots, A_{s 0} \tilde{u}_{1}\right) \in D(\mathbb{B}), \tilde{v}_{2}=\left(\tilde{u}_{2}, A_{10} \tilde{u}_{2}, \ldots, A_{s 0} \tilde{u}_{2}\right) \in D(\mathbb{B})$,

$$
\begin{aligned}
\left(\mathbb{B} \tilde{v}_{1}, \tilde{v}_{2}\right)_{\mathscr{H}} & =\left(B \tilde{u}_{1}, \tilde{u}_{2}\right)_{H}+\left(A_{12} \tilde{u}_{1}, A_{10} \tilde{u}_{2}\right)_{H^{1}}+\cdots+\left(A_{s 2} \tilde{u}_{1}, A_{s 0} \tilde{u}_{2}\right)_{H^{s}} \\
& =\left(\tilde{u}_{1}, B \tilde{u}_{2}\right)_{H}+\left(A_{10} \tilde{u}_{1}, A_{12} \tilde{u}_{2}\right)_{H^{1}}+\cdots+\left(A_{s 0} \tilde{u}_{1}, A_{s 2} \tilde{u}_{2}\right)_{H^{s}} \\
& =\left(\tilde{v}_{1}, \mathbb{B} \tilde{v}_{2}\right)_{\mathscr{H}} .
\end{aligned}
$$

Consequently, the operator $\mathbb{B}$ is symmetric. In turn, equation

$$
\lambda_{0} v+\mathbb{B} v=F, \quad F:=\left(f, f_{1}, \ldots, f_{s}\right),
$$

where $v=\left(u, A_{10} u, \ldots, A_{s 0} u\right)$, is equivalent to the system

$$
\begin{gathered}
L\left(\lambda_{0}\right) u=\lambda_{0} u+B u=f \\
L_{v}\left(\lambda_{0}\right) u=\lambda_{0} A_{\nu 0} u+A_{\nu 2} u=f_{v}, \quad v=1, \ldots, s .
\end{gathered}
$$

By virtue of condition (6), problem (2.11) has a unique solution

$$
u=\mathbb{L}\left(\lambda_{0}\right)^{-1}\left(f, f_{1}, \ldots, f_{s}\right)
$$

So, a solution of (2.10) has the following form:

$$
v=\left(\mathbb{L}\left(\lambda_{0}\right)^{-1}\left(f, f_{1}, \ldots, f_{s}\right), A_{10} \mathbb{L}\left(\lambda_{0}\right)^{-1}\left(f, f_{1}, \ldots, f_{s}\right), \ldots, A_{s 0} \mathbb{L}\left(\lambda_{0}\right)^{-1}\left(f, f_{1}, \ldots, f_{s}\right)\right) .
$$

Hence, the operator $\mathbb{B}$ is closed and the image $\operatorname{Im}\left(\lambda_{0} \mathbb{I}+\mathbb{B}\right)=\mathscr{H}$, where $\mathbb{I}$ is the identity operator in $\mathcal{H}$. By virtue of [3, Chapter $Y$, Section 3], the operator $\mathbb{B}$ is selfadjoint. From condition (5), it follows that $(\mathbb{B} v, v) \geq 0, v \in D(\mathbb{B})$. Consequently, the operator $\mathbb{B}$ is selfadjoint and nonnegative. By condition $(7), \mathbb{B}^{-1 / 2} v_{1}$ is well defined. Then, problem (2.8) has a unique solution $v(t) \in C^{2}(\mathbb{R} ; \mathscr{H}(\mathbb{B}), \mathscr{H})$ and

$$
v(t)=\cos \left(t \mathbb{B}^{1 / 2}\right) v_{0}+\sin \left(t \mathbb{B}^{1 / 2}\right) \mathbb{B}^{-1 / 2} v_{1},
$$

where $\cos \left(t \mathbb{B}^{1 / 2}\right) v=\int_{0}^{\infty} \cos \left(t \lambda^{1 / 2}\right) d E(\lambda) v, \sin \left(t \mathbb{B}^{1 / 2}\right) v=\int_{0}^{\infty} \sin \left(t \lambda^{1 / 2}\right) d E(\lambda) v$, and $E(\lambda)$ is the spectral decomposition of the selfadjoint operator $\mathbb{B}$.

Obviously, $v_{0} \in \mathscr{H}(\mathbb{B})$. Show now that $v_{1} \in \mathcal{H}\left(\mathbb{B}^{1 / 2}\right)$. From the definition of the operator $\mathbb{B}$, it follows that $\mathscr{H}(\mathbb{B}) \subset H(B) \oplus H^{1} \oplus \cdots \oplus H^{s}$. By Section 1 , $(\mathcal{H}(\mathbb{B}), \mathscr{H})_{1 / 2,2}=$ $\mathscr{H}\left(\mathbb{B}^{1 / 2}\right)$. Then,

$$
\mathscr{H}\left(\mathbb{B}^{1 / 2}\right)=(\mathscr{H}(\mathbb{B}), \mathscr{H})_{1 / 2,2} \subset(H(B), H)_{1 / 2,2} \oplus H^{1} \oplus \cdots \oplus H^{s} .
$$


Assume, first, that $v=\left(u, A_{10} u, \ldots, A_{s 0} u\right) \in D(\mathbb{B})$, that is, $u \in D(B)$. Then, by virtue of conditions (2) and (5) and the property of interpolation spaces: $\mathscr{H}(\mathbb{B}) \subset(\mathscr{H}(\mathbb{B}), \mathscr{H})_{1 / 2,2} \subset$ $\mathscr{H}$, we get

$$
\begin{aligned}
\|v\|_{\mathscr{H}\left(\mathbb{B}^{1 / 2}\right)}^{2} & =\left\|\mathbb{B}^{1 / 2} v\right\|_{\mathscr{H}}^{2}+\|v\|_{\mathscr{H}}^{2}=\left(\mathbb{B}^{1 / 2} v, \mathbb{B}^{1 / 2} v\right)_{\mathscr{H}}+(v, v)_{\mathscr{H}}=(\mathbb{B} v, v)_{\mathscr{H}}+(v, v)_{\mathscr{H}} \\
& =(B u, u)_{H}+\sum_{\nu=1}^{s}\left(A_{\nu 2} u, A_{\nu 0} u\right)_{H^{v}}+(u, u)_{H}+\sum_{\nu=1}^{s}\left(A_{\nu 0} u, A_{\nu 0} u\right)_{H^{v}} \\
& \leq C\|u\|_{(H(B), H)_{1 / 2,2}}^{2} .
\end{aligned}
$$

Now, $v_{1}:=\left(u_{1}, A_{10} u_{1}, \ldots, A_{s 0} u_{1}\right)$, where $u_{1} \in(H(B), H)_{1 / 2,2}$. Then, there exists a sequence $u^{n} \in H(B)$ such that

$$
\lim _{n \rightarrow \infty}\left\|u^{n}-u_{1}\right\|_{(H(B), H)_{1 / 2,2}}=0
$$

since the space $H(B)$ is dense in $(H(B), H)_{1 / 2,2}$. Moreover, $u^{n}$ is a fundamental sequence, that is, $\lim _{n \rightarrow \infty}\left\|u^{n}-u^{m}\right\|_{(H(B), H)_{1 / 2,2}}=0$. By $(2.16)$, we get

$$
\left\|v^{n}-v^{m}\right\|_{\mathscr{H}\left(\mathbb{B}^{1 / 2}\right)} \leq C\left\|u^{n}-u^{m}\right\|_{(H(B), H)_{1 / 2,2}},
$$

where $v^{n}=\left(u^{n}, A_{10} u^{n}, \ldots, A_{s 0} u^{n}\right)$. Therefore, the sequence $v^{n}$ is a fundamental in the Hilbert space $\mathscr{H}\left(\mathbb{B}^{1 / 2}\right)$. Hence, $v^{n}$ converges in $\mathscr{H}\left(\mathbb{B}^{1 / 2}\right)$, that is, there exists $\bar{v}=\left(\bar{u}, A_{10} \bar{u}, \ldots\right.$, $\left.A_{s 0} \bar{u}\right) \in \mathscr{H}\left(\mathbb{B}^{1 / 2}\right)$ such that $\lim _{n \rightarrow \infty}\left\|v^{n}-\bar{v}\right\|_{\mathscr{H}\left(\mathbb{B}^{1 / 2}\right)}=0$. In particular,

$$
\lim _{n \rightarrow \infty}\left\|u^{n}-\bar{u}\right\|_{(H(B), H)_{1 / 2,2}}=0
$$

Then, by virtue of (2.17), $\bar{u}=u_{1}$ and, therefore, $\bar{v}=v_{1}$. Hence, $v_{1} \in \mathscr{H}\left(\mathbb{B}^{1 / 2}\right)$. Moreover, writing (2.16) for $v^{n}$ and passing to the limit when $n \rightarrow \infty$, we get that

$$
\|v\|_{\mathscr{H}\left(\mathbb{B}^{1 / 2}\right)} \leq C\|u\|_{(H(B), H)_{1 / 2,2}}
$$

is also true for $v=\left(u, A_{10} u, \ldots, A_{s 0} u\right)$, for all $u \in(H(B), H)_{1 / 2,2}$.

Since

$$
\begin{aligned}
v^{\prime}(t) & =-\mathbb{B}^{1 / 2} \sin \left(t \mathbb{B}^{1 / 2}\right) v_{0}+\cos \left(t \mathbb{B}^{1 / 2}\right) v_{1}, \\
v^{\prime \prime}(t) & =-\mathbb{B} \cos \left(t \mathbb{B}^{1 / 2}\right) v_{0}-\mathbb{B}^{1 / 2} \sin \left(t \mathbb{B}^{1 / 2}\right) v_{1},
\end{aligned}
$$

then

$$
\begin{aligned}
\|v(t)\|+\left\|v^{\prime \prime}(t)\right\|+\|\mathbb{B} v(t)\| & \leq C\left(\left\|v_{0}\right\|+\left\|v_{1}\right\|+\left\|\mathbb{B} v_{0}\right\|+\left\|\mathbb{B}^{1 / 2} v_{1}\right\|\right) \\
& \leq C\left(\left\|v_{0}\right\|+\left\|v_{1}\right\|_{\mathscr{H}\left(\mathbb{B}^{1 / 2}\right)}+\left\|\mathbb{B} v_{0}\right\|\right), \quad t \in \mathbb{R} .
\end{aligned}
$$

From this, by (2.20) and condition (2), the statement of the theorem follows. 
104 Hyperbolic differential-operator equations on a whole axis

Consider now such a formulation of problem (2.1), which allows us to insert the firstorder derivative into (2.1a). Let $H$ and $H^{\nu}, v=1, \ldots, s$, be Hilbert spaces. Consider the following Cauchy problem (abstract initial boundary value problem):

$$
\begin{gathered}
L\left(D_{t}\right) u:=u^{\prime \prime}(t)+A u^{\prime}(t)+B u(t)=h(t), \\
L_{\nu}\left(D_{t}\right) u:=\left(A_{\nu 0} u(t)\right)^{\prime \prime}+A_{\nu 2} u(t)=h_{\nu}(t), \quad v=1, \ldots, s, \\
u(0)=u_{0}, \quad u^{\prime}(0)=u_{1},
\end{gathered}
$$

where $t \geq 0 ; A$ and $B$ are operators in $H ; A_{\nu 0}$ and $A_{\nu 2}$ are operators from a subspace of $H$ into $H^{\nu} ; u(t)$ from $[0, \infty)$ into $H$ is an unknown function; $h(t)$ and $h_{\nu}(t)$ from $[0, \infty)$ into $H$ and $H^{\nu}$, respectively, are given functions. Note that operators $A, B, A_{\nu 0}$, and $A_{\nu 2}$ are, generally speaking, unbounded.

Consider, in the Hilbert space $\mathscr{H}:=H \oplus H^{1} \oplus \cdots \oplus H^{s}$, operators $\mathbb{A}$ and $\mathbb{B}$ given by the equalities

$$
\begin{gathered}
D(\mathbb{A}):=D(A) \oplus H^{1} \oplus \cdots \oplus H^{s}, \\
\mathbb{A}\left(u, v_{1}, \ldots, v_{s}\right):=(A u, 0, \ldots, 0), \\
D(\mathbb{B}):=\left\{v \mid v:=\left(u, A_{10} u, \ldots, A_{s 0} u\right), u \in D(B)\right\}, \\
\mathbb{B}\left(u, A_{10} u, \ldots, A_{s 0} u\right):=\left(B u, A_{12} u, \ldots, A_{s 2} u\right) .
\end{gathered}
$$

THEOREM 2.2. Let the following conditions be satisfied:

(1) $B$ is an operator in a Hilbert space $H$ with a dense domain $D(B)$; $A$ is an operator in $H$ with $D(A) \supset(H(B), H)_{1 / 2,2}$; the embedding $H(B) \subset H$ is compact;

(2) the operators $A_{\nu 0}, v=1, \ldots, s$, from $(H(B), H)_{1 / 2,2}$ into $H^{v}$ act compactly, and the operators $A_{v 2}, v=1, \ldots, s$, from $H(B)$ into $H^{v}$ act boundedly;

(3) the linear manifold $\left\{v \mid v:=\left(u, A_{10} u, \ldots, A_{s 0} u\right), u \in D(B)\right\}$ is dense in the Hilbert space $H \oplus H^{1} \oplus \cdots \oplus H^{s}$;

(4) for all $u \in D(B), v \in D(B)$,

$$
\begin{aligned}
& (B u, v)_{H}+\left(A_{12} u, A_{10} v\right)_{H^{1}}+\cdots+\left(A_{s 2} u, A_{s 0} v\right)_{H^{s}} \\
& =(u, B v)_{H}+\left(A_{10} u, A_{12} v\right)_{H^{1}}+\cdots+\left(A_{s 0} u, A_{s 2} v\right)_{H^{s}}
\end{aligned}
$$

(5) for all $u \in D(B)$ and some $C, c \neq 0$,

$$
\begin{aligned}
C\|u\|_{(H(B), H)_{1 / 2,2}}^{2} & \geq(B u, u)_{H}+\left(A_{12} u, A_{10} u\right)_{H^{1}}+\cdots+\left(A_{s 2} u, A_{s 0} u\right)_{H^{s}} \\
& \geq c^{2}\left(\|u\|_{H}^{2}+\left\|A_{10} u\right\|_{H^{1}}^{2}+\cdots+\left\|A_{s 0} u\right\|_{H^{s}}^{2}\right) ;
\end{aligned}
$$

(6) some real number $\lambda_{0}$ is a regular point for the operator pencil $\mathbb{L}(\lambda): u \rightarrow \mathbb{L}(\lambda) u:=$ $\left((\lambda I+B) u,\left(\lambda A_{10}+A_{12}\right) u, \ldots,\left(\lambda A_{s 0}+A_{s 2}\right) u\right)$, which acts boundedly from $H(B)$ onto $H \oplus H^{1} \oplus \cdots \oplus H^{s}$;

(7) $A$ is a skew-symmetric operator in $H$, that is, $A^{*} u=-A u, u \in D(A)$, and $A$ from $(H(B), H)_{1 / 2,2}$ into $H$ is bounded; 
(8) $h \in W_{p}^{1}((0, \infty) ; H) \cap L_{1}((0, \infty) ; H), h_{\nu} \in W_{p}^{1}\left((0, \infty) ; H^{v}\right) \cap L_{1}\left((0, \infty) ; H^{v}\right), v=1,2$, for some $p>1$;

(9) $u_{0} \in H(B), u_{1} \in(H(B), H)_{1 / 2,2}$.

Then there exists a unique solution $u(t)$ of problem (2.23) such that the function $t \rightarrow$ $\left(u(t), A_{10} u(t), \ldots, A_{s 0} u(t)\right)$ from $[0, \infty)$ into $H \oplus H^{1} \oplus \cdots \oplus H^{s}$ is twice continuously differentiable and from $[0, \infty)$ into $H(B) \oplus H^{1} \oplus \cdots \oplus H^{s}$ is continuous, and for the solution the following estimate holds:

$$
\begin{aligned}
& \|u(t)\|_{(H(B), H)_{1 / 2,2}}+\left\|u^{\prime}(t)\right\|+\sum_{\nu=1}^{s}\left\|\left(A_{\nu 0} u(t)\right)^{\prime}\right\|_{H^{\nu}} \\
& \quad \leq C\left(\left\|u_{0}\right\|_{(H(B), H)_{1 / 2,2}}+\left\|u_{1}\right\|_{(H(B), H)_{1 / 2,2}}+\|h\|_{L_{1}((0, \infty) ; H)}+\sum_{\nu=1}^{2}\left\|h_{\nu}\right\|_{L_{1}\left((0, \infty) ; H^{\nu}\right)}\right), \quad \forall t \geq 0,
\end{aligned}
$$

consequently (since $\left.H(B) \subset(H(B), H)_{1 / 2,2} \subset H\right)$, problem (2.23) is stable.

Note that substituting $t=-\tau, \tau \geq 0$, one can consider problem (2.23) for $t \leq 0$ too. Therefore, in fact, Theorem 2.2 is true for $t \in \mathbb{R}$.

Proof. Consider, in the Hilbert space $\mathscr{H}:=H \oplus H^{1} \oplus \cdots \oplus H^{s}$, the above-mentioned operators $\mathbb{A}$ and $\mathbb{B}$. Then the Cauchy problem

$$
\begin{aligned}
v^{\prime \prime}(t)+\mathbb{A} v^{\prime}(t)+\mathbb{B} v(t) & =f(t), \\
v(0)=v_{0}, \quad v^{\prime}(0) & =v_{1},
\end{aligned}
$$

is equivalent to the Cauchy problem (2.23), where $v_{0}:=\left(u_{0}, A_{10} u_{0}, \ldots, A_{s 0} u_{0}\right), v_{1}:=\left(u_{1}\right.$, $\left.A_{10} u_{1}, \ldots, A_{s 0} u_{1}\right), f(t):=\left(h(t), h_{1}(t), \ldots, h_{s}(t)\right)$, and $v(t):=\left(u(t), A_{10} u(t), \ldots, A_{s 0} u(t)\right)$ (for the proof, see the proof of Theorem 2.1).

Apply Theorem A.1 (see the appendix) to problem (2.28), where $\tilde{A}:=\mathbb{A}, \tilde{B}:=\mathbb{B}$. It was proved in Theorem 2.1 that the operator $\mathbb{B}$ is selfadjoint.

From condition (5), it follows that $(\mathbb{B} v, v) \geq c^{2}(v, v), v \in D(\mathbb{B})$. Consequently, the operator $\mathbb{B}$ is selfadjoint and positive-definite. So, by conditions (1), (2), and (3), conditions (1) and (2) of Theorem A.1 are fulfilled.

From the proof of Theorem 2.1, it follows that $\mathscr{H}\left(\mathbb{B}^{1 / 2}\right) \subset(H(B), H)_{1 / 2,2} \oplus H^{1} \oplus \cdots \oplus$ $H^{s}$. This implies, by conditions $(1)$ and $(7), D(\mathbb{A}) \supset D\left(\mathbb{B}^{1 / 2}\right)$, the operator $\mathbb{A}$ from $\mathscr{H}\left(\mathbb{B}^{1 / 2}\right)$ into $\mathcal{H}$ is bounded and is skew-symmetric. Hence, condition (3) of Theorem A.1 is satisfied. From condition (8), it follows condition (4) of Theorem A.1. Similar arguments to those in the proof of Theorem 2.1 gives us that $v_{0} \in \mathscr{H}(\mathbb{B}), v_{1} \in \mathscr{H}\left(\mathbb{B}^{1 / 2}\right)$, that is, the last condition (5) of Theorem A.1 is satisfied too. So, on each interval $[0, T]$, we have a unique solution $v(t) \in C^{2}\left([0, T] ; \mathscr{H}(\mathbb{B}), \mathscr{H}\left(\mathbb{B}^{1 / 2}\right), \mathscr{H}\right)$ of problem $(2.28)$. In order to get the estimate of Theorem 2.2, one should use the proof of Theorem A.1 from the appendix. In particular, it follows from the proof that

$$
\left(\begin{array}{c}
v(t) \\
v^{\prime}(t)
\end{array}\right)=e^{t \mathscr{A}}\left(\begin{array}{c}
v_{0} \\
v_{1}
\end{array}\right)+\int_{0}^{t} e^{(t-\tau) \mathscr{A}}\left(\begin{array}{c}
0 \\
f(\tau)
\end{array}\right) d \tau
$$


where $\mathscr{A}=\left(\begin{array}{cc}0 & I \\ -\mathbb{B} & -\mathbb{A}\end{array}\right)$. Moreover,

$$
\|v(t)\|_{\mathscr{H}\left(\mathbb{B}^{1 / 2}\right)}+\left\|v^{\prime}(t)\right\|_{\mathscr{H}} \leq\left\|v_{0}\right\|_{\mathscr{H}\left(\mathbb{B}^{1 / 2}\right)}+\left\|v_{1}\right\|_{\mathscr{H}}+\int_{0}^{t}\|f(\tau)\|_{\mathscr{H}} d \tau .
$$

Using conditions (2), (8), and (9), and the inequality (2.20), we get the estimate of the theorem.

\section{Initial boundary value problems for hyperbolic equations}

Consider, in the domain $\mathbb{R} \times[0,1]$, an initial boundary value problem for the hyperbolic equation

$$
\begin{gathered}
L\left(D_{t}\right) u:=D_{t t}^{2} u(t, x)-D_{x}\left(b(x) D_{x} u(t, x)\right)=0, \quad(t, x) \in \mathbb{R} \times[0,1], \\
L_{1}\left(D_{t}\right) u:=\alpha D_{t t}^{2}[u(t, 0)]+D_{x} u(t, 0)=0, \quad t \in \mathbb{R}, \\
L_{2}\left(D_{t}\right) u:=\beta D_{t t}^{2}[u(t, 1)]+D_{x} u(t, 1)=0, \quad t \in \mathbb{R}, \\
u(0, x)=u_{0}(x), \quad D_{t} u(0, x)=u_{1}(x), \quad x \in[0,1],
\end{gathered}
$$

where $\alpha, \beta$ are real numbers, $D_{t}:=\partial / \partial t$ and $D_{x}:=\partial / \partial x$.

This problem was considered, by a different approach, in [1]. As it was mentioned in [1], "physically, such a problem may represent the longitudinal displacements of an inhomogeneous rod under the action of forces at the two ends which are proportional to the acceleration. In particular, this situation is realized if there are massive loads at the ends (see, e.g., [2, Chapter 12]) and in this case we have $\alpha<0$ and $\beta>0$."

Theorem 3.1. Let the following conditions be satisfied:

(1) $b \in C^{1}[0,1] ; b(x)>0$ for $x \in[0,1]$;

(2) $\alpha<0, \beta>0$;

(3) $u_{0} \in W_{2}^{2}(0,1), u_{1} \in W_{2}^{1}(0,1)$;

(4) $\int_{0}^{1} u_{1}(x) d x-\alpha b(0) u_{1}(0)+\beta b(1) u_{1}(1)=0$.

Then there exists a unique solution $u(t, x)$ of problem (3.1) such that the function $t \rightarrow$ $(u(t, x), u(t, 0), u(t, 1))$ from $\mathbb{R}$ into $L_{2}(0,1) \oplus \mathbb{C} \oplus \mathbb{C}$ is twice continuously differentiable, and from $\mathbb{R}$ into $W_{2}^{2}(0,1) \oplus \mathbb{C} \oplus \mathbb{C}$ is continuous, and for $t \in \mathbb{R}$ the following estimate holds:

$$
\begin{aligned}
& \|u(t, \cdot)\|_{L_{2}(0,1)}+\left\|D_{t t}^{2} u(t, \cdot)\right\|_{L_{2}(0,1)}+\left|D_{t t}^{2}[u(t, 0)]\right|+\left|D_{t t}^{2}[u(t, 1)]\right| \\
& \quad+\left\|D_{x x}^{2} u(t, \cdot)\right\|_{L_{2}(0,1)} \leq C\left(\left\|u_{0}\right\|_{W_{2}^{2}(0,1)}+\left\|u_{1}\right\|_{W_{2}^{1}(0,1)}\right)
\end{aligned}
$$

consequently, problem (3.1) is stable.

Proof. Apply Theorem 2.1. Consider, in the Hilbert space $H:=L_{2}(0,1)$, an operator $B$ given by the equalities

$$
D(B):=W_{2}^{2}(0,1), \quad B u:=-\left(b(x) u^{\prime}(x)\right)^{\prime} .
$$


Taking $H^{1}:=-(b(0) / \alpha) \mathbb{C}, H^{2}:=(b(1) / \beta) \mathbb{C}$, and

$$
\begin{array}{ll}
A_{10} u:=\alpha u(0), & A_{20} u:=\beta u(1), \\
A_{12} u:=u^{\prime}(0), & A_{22} u:=u^{\prime}(1),
\end{array}
$$

problem (3.1) can be rewritten in the form (2.1), where $u(t):=u(t, \cdot)$ is a function with values in the Hilbert space $H:=L_{2}(0,1)$, and $u_{0}:=u_{0}(\cdot), u_{1}:=u_{1}(\cdot)$.

From [4, Section 3.2.5], it follows that condition (1) of Theorem 2.1 is satisfied. From $\left[4\right.$, Section 4.3.1], it follows that $\left(W_{2}^{2}(0,1), L_{2}(0,1)\right)_{1 / 2,2}=W_{2}^{1}(0,1)$. Then condition (2) of Theorem 2.1 is satisfied too. Condition (3) of Theorem 2.1 follows from Theorem A.2 (see the appendix). We prove conditions (4) and (5) of Theorem 2.1. For $u_{1} \in W_{2}^{2}(0,1)$, $u_{2} \in W_{2}^{2}(0,1)$, we have

$$
\begin{aligned}
\left(B u_{1}, u_{2}\right)_{L_{2}(0,1)}+\left(A_{12} u_{1}, A_{10} u_{2}\right)_{-(b(0) / \alpha) \mathbb{C}}+\left(A_{22} u_{1}, A_{20} u_{2}\right)_{(b(1) / \beta) \mathbb{C}} \\
=-\int_{0}^{1} \frac{d}{d x}\left(b(x) \frac{d u_{1}(x)}{d x}\right) \overline{u_{2}(x)} d x-\frac{b(0)}{\alpha} u_{1}^{\prime}(0) \cdot \overline{\alpha u_{2}(0)}+\frac{b(1)}{\beta} u_{1}^{\prime}(1) \overline{\beta u_{2}(1)} \\
=-\int_{0}^{1} u_{1}(x) \frac{d}{d x}\left(\overline{b(x) \frac{d u_{2}(x)}{d x}}\right) d x-\left.b(x) u_{1}^{\prime}(x) \overline{u_{2}(x)}\right|_{0} ^{1} \\
+\left.u_{1}(x)\left(\overline{b(x) u_{2}^{\prime}(x)}\right)\right|_{0} ^{1}-b(0) u_{1}^{\prime}(0) \overline{u_{2}(0)}+b(1) u_{1}^{\prime}(1) \overline{u_{2}(1)} \\
=\left(u_{1}, B u_{2}\right)_{L_{2}(0,1)}+\left(A_{10} u_{1}, A_{12} u_{2}\right)_{-(b(0) / \alpha) \mathbb{C}}+\left(A_{20} u_{1}, A_{22} u_{2}\right)_{(b(1) / \beta) \mathbb{C}}
\end{aligned}
$$

that is, condition (4) of Theorem 2.1 is satisfied. For $u \in W_{2}^{2}(0,1)$, we have

$$
\begin{aligned}
& (B u, u)_{L_{2}(0,1)}+\left(A_{12} u, A_{10} u\right)_{-(b(0) / \alpha) \mathbb{C}}+\left(A_{22} u, A_{20} u\right)_{(b(1) / \beta) \mathbb{C}} \\
& =\int_{0}^{1} b(x)\left|u^{\prime}(x)\right|^{2} d x-\left.b(x) u^{\prime}(x) \overline{u(x)}\right|_{0} ^{1}-b(0) u^{\prime}(0) \overline{u(0)}+b(1) u^{\prime}(1) \overline{u(1)} \\
& \quad=\int_{0}^{1} b(x)\left|u^{\prime}(x)\right|^{2} d x \geq 0 .
\end{aligned}
$$

On the other hand, $\int_{0}^{1} b(x)\left|u^{\prime}(x)\right|^{2} d x \leq C\|u\|_{W_{2}^{1}(0,1)}^{2}$, that is, condition (5) of Theorem 2.1 is satisfied too. Denote

$$
\begin{aligned}
L(\lambda) u & :=(\lambda I+B) u=\lambda u(x)-\left(b(x) u^{\prime}(x)\right)^{\prime}, \\
L_{1}(\lambda) u & :=\left(\lambda A_{10}+A_{12}\right) u=\lambda \alpha u(0)+u^{\prime}(0), \\
L_{2}(\lambda) u & :=\left(\lambda A_{20}+A_{22}\right) u=\lambda \beta u(1)+u^{\prime}(1) .
\end{aligned}
$$

From Theorem A.3 (see the appendix), it follows that for any $\varepsilon>0$, there exists $R_{\varepsilon}>0$ such that for all complex numbers $\lambda$ which satisfy $|\lambda|>R_{\varepsilon}$ and lying inside the angle

$$
-\pi+\varepsilon<\arg \lambda<\pi-\varepsilon
$$


the operator $\mathbb{L}(\lambda): u \rightarrow \mathbb{L}(\lambda) u:=\left(L(\lambda) u, L_{1}(\lambda) u, L_{2}(\lambda) u\right)$ from $W_{2}^{2}(0,1)$ onto $L_{2}(0,1) \oplus$ $-(b(0) / \alpha) \mathbb{C} \oplus(b(1) / \beta) \mathbb{C}$ is an isomorphism, that is, condition (6) of Theorem 2.1 is satisfied. Condition (7) of Theorem 2.1 is fulfilled in view of condition (4) (see for details [1]). Condition (8) of Theorem 2.1 follows from condition (3). So, for problem (3.1), all conditions of Theorem 2.1 are fulfilled and the statement of Theorem 3.1 follows.

We present now an application of Theorem 2.2. Consider, in the domain $[0, \infty) \times$ $[0,1]$, an initial boundary value problem for the hyperbolic equation

$$
\begin{gathered}
L\left(D_{t}\right) u:=D_{t t}^{2} u(t, x)+i a(x) D_{t} u(t, x)-D_{x}\left(b(x) D_{x} u(t, x)\right)+c(x) u(t, x) \\
=h(t, x), \quad(t, x) \in[0, \infty) \times[0,1], \\
L_{1}\left(D_{t}\right) u:=\alpha D_{t t}^{2}[u(t, 0)]+D_{x} u(t, 0)=h_{1}(t), \quad t \in[0, \infty), \\
L_{2}\left(D_{t}\right) u:=\beta D_{t t}^{2}[u(t, 1)]+D_{x} u(t, 1)=h_{2}(t), \quad t \in[0, \infty), \\
u(0, x)=u_{0}(x), \quad D_{t} u(0, x)=u_{1}(x), \quad x \in[0,1],
\end{gathered}
$$

where $\alpha, \beta$ are real numbers, $i=\sqrt{-1}, D_{t}:=\partial / \partial t, D_{x}:=\partial / \partial x$.

THeOrem 3.2. Let the following conditions be satisfied:

(1) $a \in C[0,1]$ and is real-valued; $b \in C^{1}[0,1], b(x)>0$ for $x \in[0,1] ; c \in C[0,1], c(x)>$ 0 for $x \in[0,1]$;

(2) $\alpha<0, \beta>0$;

(3) $h \in W_{p}^{1}\left((0, \infty) ; L_{2}(0,1)\right) \cap L_{1}\left((0, \infty) ; L_{2}(0,1)\right), h_{v} \in W_{p}^{1}(0, \infty) \cap L_{1}(0, \infty), v=1,2$, for some $p>1$;

(4) $u_{0} \in W_{2}^{2}(0,1), u_{1} \in W_{2}^{1}(0,1)$.

Then there exists a unique solution $u(t, x)$ of problem (3.9) such that the function $t \rightarrow$ $(u(t, x), u(t, 0), u(t, 1))$ from $[0, \infty)$ into $L_{2}(0,1) \oplus \mathbb{C} \oplus \mathbb{C}$ is twice continuously differentiable, and from $[0, \infty)$ into $W_{2}^{2}(0,1) \oplus \mathbb{C} \oplus \mathbb{C}$ is continuous, and for the solution the following estimate holds:

$$
\begin{aligned}
& \|u(t, \cdot)\|_{L_{2}(0,1)}+\left\|D_{t} u(t, \cdot)\right\|_{L_{2}(0,1)}+\left|D_{t}[u(t, 0)]\right|+\left|D_{t}[u(t, 1)]\right| \\
& \quad \leq C\left(\left\|u_{0}\right\|_{W_{2}^{1}(0,1)}+\left\|u_{1}\right\|_{W_{2}^{1}(0,1)}+\int_{0}^{\infty}\|h(t, \cdot)\|_{L_{2}(0,1)} d t+\sum_{\nu=1}^{2} \int_{0}^{\infty}\left|h_{\nu}(t)\right| d t\right), \quad \forall t \geq 0,
\end{aligned}
$$

consequently, problem (3.9) is stable.

Note that this theorem, as Theorem 2.2, is also true for $t \leq 0$ and, therefore, for $t \in \mathbb{R}$.

Proof. Apply Theorem 2.2. Consider, in the Hilbert space $H:=L_{2}(0,1)$, operators $A$ and $B$ given by the equalities

$$
\begin{gathered}
D(A):=L_{2}(0,1), \quad A u:=i a(x) u(x), \\
D(B):=W_{2}^{2}(0,1), \quad B u:=-\left(b(x) u^{\prime}(x)\right)^{\prime}+c(x) u(x) .
\end{gathered}
$$


Taking $H^{1}:=-(b(0) / \alpha) \mathbb{C}, H^{2}:=(b(1) / \beta) \mathbb{C}$, and $A_{10} u:=\alpha u(0), A_{20} u:=\beta u(1), A_{12} u:=$ $u^{\prime}(0), A_{22} u:=u^{\prime}(1)$, problem (3.9) can be rewritten in the form (2.23), where $u(t):=$ $u(t, \cdot)$ and $h(t):=h(t, \cdot)$ are functions with values in the Hilbert space $H:=L_{2}(0,1)$, and $\varphi_{0}:=\varphi_{0}(\cdot), \varphi_{1}:=\varphi_{1}(\cdot)$.

From [4, Section 3.2.5], it follows that $D(B)$ is dense in $H$, and the embedding $H(B) \subset$ $H$ is compact, and from $\left[4\right.$, Section 4.3.1] it follows that $\left(W_{2}^{2}(0,1), L_{2}(0,1)\right)_{1 / 2,2}=W_{2}^{1}(0,1)$. Therefore, condition (1) of Theorem 2.2 is satisfied. It is well known that the embedding $W_{2}^{k}(0,1) \subset C^{m}[0,1], k>m \geq 0$, is compact (see, e.g., [4, Section 4.10.2, formula (15)]). Then condition (2) of Theorem 2.2 is satisfied too. Condition (3) of Theorem 2.2 follows from Theorem A.2 (see the appendix). We prove conditions (4) and (5) of Theorem 2.2. For $u \in W_{2}^{2}(0,1), v \in W_{2}^{2}(0,1)$, we have

$$
\begin{aligned}
&(B u, v)_{L_{2}(0,1)}+\left(A_{12} u, A_{10} v\right)_{-(b(0) / \alpha) \mathbb{C}}+\left(A_{22} u, A_{20} v\right)_{(b(1) / \beta) \mathbb{C}} \\
&=-\int_{0}^{1} \frac{d}{d x}\left(b(x) \frac{d u(x)}{d x}\right) \overline{v(x)} d x+\int_{0}^{1} c(x) u(x) \overline{v(x)} d x \\
&- \frac{b(0)}{\alpha} u^{\prime}(0) \overline{\alpha v(0)}+\frac{b(1)}{\beta} u^{\prime}(1) \overline{\beta v(1)} \\
&=-\int_{0}^{1} u(x) \frac{d}{d x}\left(\overline{b(x)} \frac{d v(x)}{d x}\right) d x+\int_{0}^{1} c(x) u(x) \overline{v(x)} d x-\left.b(x) u^{\prime}(x) \overline{v(x)}\right|_{0} ^{1} \\
&+\left.u(x)\left(\overline{b(x) v^{\prime}(x)}\right)\right|_{0} ^{1}-b(0) u^{\prime}(0) \overline{v(0)}+b(1) u^{\prime}(1) \overline{v(1)} \\
&=(u, B v)_{L_{2}(0,1)}+\left(A_{10} u, A_{12} v\right)_{-(b(0) / \alpha) \mathbb{C}}+\left(A_{20} u, A_{22} v\right)_{(b(1) / \beta) \mathbb{C}}
\end{aligned}
$$

that is, condition (4) of Theorem 2.2 is satisfied. For $u \in W_{2}^{2}(0,1)$, using conditions (1) and (2) and that $W_{2}^{1}(0,1) \subset C[0,1]$ is bounded, we have

$$
\begin{aligned}
&(B u, u)_{L_{2}(0,1)}+\left(A_{12} u, A_{10} u\right)_{-(b(0) / \alpha) \mathbb{C}}+\left(A_{22} u, A_{20} u\right)_{(b(1) / \beta) \mathbb{C}} \\
&=\int_{0}^{1} b(x)\left|u^{\prime}(x)\right|^{2} d x+\int_{0}^{1} c(x)|u(x)|^{2} d x-\left.b(x) u^{\prime}(x) \overline{u(x)}\right|_{0} ^{1} \\
&-b(0) u^{\prime}(0) \overline{u(0)}+b(1) u^{\prime}(1) \overline{u(1)} \\
&= \int_{0}^{1} b(x)\left|u^{\prime}(x)\right|^{2} d x+\int_{0}^{1} c(x)|u(x)|^{2} d x \\
& \geq \frac{1}{\sqrt{2}} \min \left\{\min _{x \in[0,1]} b(x), \min _{x \in[0,1]} c(x)\right\}\|u\|_{W_{2}^{1}(0,1)}^{2} \\
& \geq c^{2}\left(\|u\|_{L_{2}(0,1)}^{2}-b(0) \alpha|u(0)|^{2}+b(1) \beta|u(1)|^{2}\right) \\
&= c^{2}\left(\|u\|_{L_{2}(0,1)}^{2}+\left\|A_{10} u\right\|_{-(b(0) / \alpha) \mathbb{C}}^{2}+\left\|A_{20} u\right\|_{(b(1) / \beta) \mathbb{C}}^{2}\right), \quad \exists c \neq 0 .
\end{aligned}
$$

On the other hand, $\int_{0}^{1} b(x)\left|u^{\prime}(x)\right|^{2} d x+\int_{0}^{1} c(x)|u(x)|^{2} d x \leq C\|u\|_{W_{2}^{1}(0,1)}^{2}$, that is, condition (5) of Theorem 2.2 is satisfied too. Condition (6) of Theorem 2.2 is checked as in 
110 Hyperbolic differential-operator equations on a whole axis

the proof of Theorem 3.1. We check condition (7) of Theorem 2.2. Take $u, v \in D(A)=$ $L_{2}(0,1)$. Then,

$$
\begin{aligned}
(A u, v)_{L_{2}(0,1)} & =\int_{0}^{1} i a(x) u(x) \overline{v(x)} d x=-\int_{0}^{1} u(x) \overline{i a(x) v(x)} d x \\
& =(u,-A v)_{L_{2}(0,1)} .
\end{aligned}
$$

Conditions (8) and (9) of Theorem 2.2 are trivial. So, for problem (3.9), all conditions of Theorem 2.2 are fulfilled and the statement of Theorem 3.2 follows.

\section{Appendix}

Consider, in a Hilbert space $H$, the Cauchy problem for the second-order hyperbolic differential-operator equation

$$
\begin{gathered}
L(D) u:=u^{\prime \prime}(t)+\tilde{A} u^{\prime}(t)+\tilde{B} u(t)=f(t), \quad t \in[0, T], \\
u(0)=g_{0}, \quad u^{\prime}(0)=g_{1},
\end{gathered}
$$

and the characteristic operator pencil

$$
L(\lambda):=\lambda^{2}+\lambda \tilde{A}+\tilde{B}
$$

Theorem A.1 (see [7, Theorem 6.4.3]). Let the following conditions be satisfied:

(1) $\tilde{B}$ is a selfadjoint positive-definite operator in a Hilbert space $H$;

(2) the embedding $H(\tilde{B}) \subset H$ is compact;

(3) $\tilde{A}$ is a skew-symmetric operator in $H$, that is, $\tilde{A}^{*} u=-\tilde{A} u, u \in D(\tilde{A})$; the operator $\tilde{A}$ from $H\left(\tilde{B}^{1 / 2}\right)$ into $H$ is bounded;

(4) $f \in W_{p}^{1}((0, T)$; $H)$, where $p>1$;

(5) $g_{0} \in D(\tilde{B}), g_{1} \in D\left(\tilde{B}^{1 / 2}\right)$.

Then, problem (A.1) has a unique solution $u \in C^{2}\left([0, T] ; H(\tilde{B}), H\left(\tilde{B}^{1 / 2}\right), H\right)$, and the solution can be expanded to the series

$$
\begin{aligned}
u(t)=\sum_{k=1}^{\infty} & \frac{e^{\lambda_{k} t}}{\left\|\tilde{B}^{1 / 2} u_{k}\right\|^{2}+\left|\lambda_{k}\right|^{2}\left\|u_{k}\right\|^{2}} \\
& \times\left(\left(\tilde{B} g_{0}-\lambda_{k} g_{1}, u_{k}\right)-\lambda_{k} \int_{0}^{t} e^{-\lambda_{k} \tau}\left(f(\tau), u_{k}\right) d \tau\right) u_{k}
\end{aligned}
$$

where $\lambda_{k}$ are purely imaginary eigenvalues and $u_{k}$ are the corresponding eigenvectors of operator pencil (A.2), and the series converges in the sense of the space $C^{2}\left([0, T] ; H(\tilde{B}), H\left(\tilde{B}^{1 / 2}\right), H\right)$.

Denote

$$
A_{\nu 0} u:=\alpha_{\nu} u^{\left(m_{\nu}\right)}(0)+\beta_{\nu} u^{\left(m_{\nu}\right)}(1)+\sum_{j=1}^{N_{\nu}} \delta_{\nu j} u^{\left(m_{\nu}\right)}\left(x_{\nu j}\right)+T_{\nu} u, \quad v=1, \ldots, m
$$


Theorem A.2 (see [7, Theorem 3.6.2]). Let the following conditions be satisfied:

(1) $m \geq 1, m_{v} \geq 0,0 \leq s \leq m$;

(2) a system of functionals (A.4) is p-regular with respect to a system of numbers $\omega_{j}:=$ $e^{2 \pi i((j-1) / m)}, j=1, \ldots, m$, that is,

$$
\left|\begin{array}{cccccc}
\alpha_{1} \omega_{1}^{m_{1}} & \cdots & \alpha_{1} \omega_{p}^{m_{1}} & \beta_{1} \omega_{p+1}^{m_{1}} & \cdots & \beta_{1} \omega_{m}^{m_{1}} \\
\vdots & \vdots & \vdots & \vdots & \vdots & \vdots \\
\vdots & \vdots & \vdots & \vdots & \vdots & \vdots \\
\alpha_{m} \omega_{1}^{m_{m}} & \cdots & \alpha_{m} \omega_{p}^{m_{m}} & \beta_{m} \omega_{p+1}^{m_{m}} & \cdots & \beta_{m} \omega_{m}^{m_{m}}
\end{array}\right| \neq 0 \text {, }
$$

where $p=m / 2$ if $m$ is even, $p=[m / 2]$ or $p=[m / 2]+1$ if $m$ is odd, $x_{v j} \in(0,1)$ and, for some $q \in(1, \infty)$, functionals $T_{v}$ in $W_{q}^{m_{v}}(0,1)$ are continuous.

Then, the linear manifold

$$
\left\{(u, v) \mid u \in C^{\infty}[0,1], A_{v 0} u=0, v=s+1, \ldots, m, v:=\left(A_{10} u, \ldots, A_{s 0} u\right)\right\},
$$

is dense in the space $W_{q}^{\ell}(0,1)+\mathbb{C}^{s}, \ell \leq \min \left\{m_{\nu}\right\}$.

Consider a principally boundary value problem for an ordinary differential equation with a variable coefficient in case when the spectral parameter appears linearly in the equation and can appear in boundary-functional conditions

$$
\begin{gathered}
L(\lambda) u:=\lambda u(x)+a(x) u^{(m)}(x)+\left.B u\right|_{x}=f(x), \quad x \in(0,1), \\
L_{\nu}(\lambda) u:=\lambda\left(\alpha_{\nu} u^{\left(m_{\nu}\right)}(0)+\beta_{\nu} u^{\left(m_{\nu}\right)}(1)+\sum_{j=1}^{N_{\nu}} \delta_{\nu j} u^{\left(m_{\nu}\right)}\left(x_{\nu j}\right)+T_{\nu} u\right) \\
+T_{\nu 0} u=g_{\nu}, \quad v=1, \ldots, s, \\
L_{\nu} u:=\alpha_{\nu} u^{\left(m_{\nu}\right)}(0)+\beta_{\nu} u^{\left(m_{\nu}\right)}(1)+\sum_{j=1}^{N_{\nu}} \delta_{\nu j} u^{\left(m_{\nu}\right)}\left(x_{\nu j}\right)+T_{\nu} u=0, \quad v=s+1, \ldots, m,
\end{gathered}
$$

where $m \geq 1, m_{v} \leq m-1, x_{\nu j} \in(0,1), 0 \leq s \leq m, B$ is an operator in $L_{2}(0,1), T_{\nu}$ and $T_{\nu 0}$ are functionals in $L_{2}(0,1)$.

Theorem A.3 (see [6]). Let the following conditions be satisfied:

(1) $m \geq 1 ; m_{v} \leq m-1 ; 0 \leq s \leq m$;

(2) $a \in C[0,1] ; a(x) \neq 0 ; a(0)=a(1)$; $\sup _{x \in[0,1]} \arg a(x)-\inf _{x \in[0,1]} \arg a(x)<2 \pi$ if $m$ is even; $\sup _{x \in[0,1]} \arg a(x)-\inf _{x \in[0,1]} \arg a(x)<\pi$ if $m$ is odd;

(3) for all $\varepsilon>0$,

$$
\|B u\|_{L_{2}(0,1)} \leq \varepsilon\|u\|_{W_{2}^{m}(0,1)}+C(\varepsilon)\|u\|_{L_{2}(0,1)}, \quad u \in W_{2}^{m}(0,1) ;
$$


112 Hyperbolic differential-operator equations on a whole axis

(4) functionals $T_{\nu}$ in $W_{2}^{m_{\nu}}(0,1)$ and functionals $T_{\nu 0}$ in $W_{2}^{m-\varepsilon}(0,1)$, for some $\varepsilon>0$, are continuous;

(5) system (A.4) is p-regular with respect to a system of numbers $\omega_{j}=e^{2 \pi i((j-1) / m)}, j=$ $1, \ldots, m$ (see Theorem A.2).

Then for any $\varepsilon>0$, there exists $R_{\varepsilon}>0$ such that for all complex numbers $\lambda$ which satisfy $|\lambda|>R_{\varepsilon}$ and for $m=2 p$ lying inside the angle,

$$
\frac{\pi m}{2}-\pi+\sup _{x \in[0,1]} \arg a(x)+\varepsilon<\arg \lambda<\frac{\pi m}{2}+\pi+\inf _{x \in[0,1]} \arg a(x)-\varepsilon,
$$

for $m=2 p+1$ lying inside the angle,

$$
\frac{\pi m}{2}+\sup _{x \in[0,1]} \arg a(x)+\varepsilon<\arg \lambda<\frac{\pi m}{2}+\pi+\inf _{x \in[0,1]} \arg a(x)-\varepsilon,
$$

and for $m=2 p-1$ lying inside the angle,

$$
\frac{\pi m}{2}-\pi+\sup _{x \in[0,1]} \arg a(x)+\varepsilon<\arg \lambda<\frac{\pi m}{2}+\inf _{x \in[0,1]} \arg a(x)-\varepsilon
$$

the operator $\mathbb{L}(\lambda): u \rightarrow \mathbb{L}(\lambda) u:=\left(L(\lambda) u, L_{1}(\lambda) u, \ldots, L_{s}(\lambda) u\right)$ from $W_{2}^{m}\left((0,1) ; L_{\nu} u=0, v=\right.$ $s+1, \ldots, m)$ onto $L_{2}(0,1) \dot{+} \mathbb{C}^{s}$ is an isomorphism, and for these $\lambda$ for a solution of problem (A.7), the estimate

$$
\|u\|_{W_{2}^{m}(0,1)}+|\lambda|\left(\|u\|_{L_{2}(0,1)}+\sum_{\nu=1}^{s}\left|A_{\nu 0} u\right|\right) \leq C(\varepsilon)\left(\|f\|_{L_{2}(0,1)}+\sum_{\nu=1}^{s}\left|g_{\nu}\right|\right)
$$

is valid, where $A_{\nu 0}$ is defined by (A.4).

Note that if boundary-functional conditions (A.7b) and (A.7c) are principally local, that is, $\alpha_{v}=0$ or $\beta_{v}=0$ for all $v=1, \ldots, m$, then the condition $a(0)=a(1)$ should be omitted.

\section{Acknowledgment}

The author was supported by the Israel Ministry of Absorption.

\section{References}

[1] P. Lancaster, A. Shkalikov, and Q. Ye, Strongly definitizable linear pencils in Hilbert space, Integral Equations Operator Theory 17 (1993), no. 3, 338-360.

[2] C. C. Lin and L. A. Segel, Mathematics Applied to Deterministic Problems in the Natural Sciences, Macmillan Publishing, New York, 1974.

[3] K. Maurin, Methods of Hilbert Spaces, Monografie Matematyczne, vol. 45, Państwowe Wydawnictwo Naukowe, Warsaw, 1967, translated from Polish by Andrzej Alexiewicz and Waclaw Zawadowski.

[4] H. Triebel, Interpolation Theory, Function Spaces, Differential Operators, North-Holland Mathematical Library, vol. 18, North-Holland, Amsterdam, 1978.

[5] S. Yakubov and Ya. Yakubov, An initial boundary value problem for hyperbolic diferentialoperator equations on a finite interval, accepted in Differential and Integral Equations. 
[6] _ Abel basis of root functions of regular boundary value problems, Math. Nachr. 197 (1999), 157-187.

[7] _ Differential-Operator Equations. Ordinary and Partial Differential Equations, Chapman \& Hall/CRC Monographs and Surveys in Pure and Applied Mathematics, vol. 103, Chapman \& Hall/CRC, Boca Raton, 2000.

[8] Ya. Yakubov, Almost periodic solutions and oscillations decay for hyperbolic differential-operator equations, Funct. Differ. Equ. 10 (2003), no. 1-2, 315-330.

Yakov Yakubov: Raymond and Beverly Sackler Faculty of Exact Sciences, School of Mathematical Sciences, Tel-Aviv University, Ramat-Aviv 69978, Israel

E-mail address: yakubov@post.tau.ac.il 


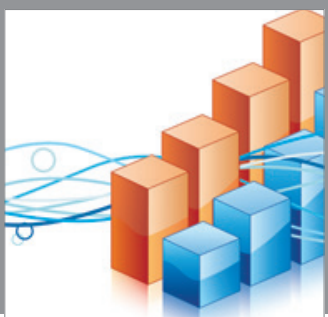

Advances in

Operations Research

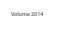

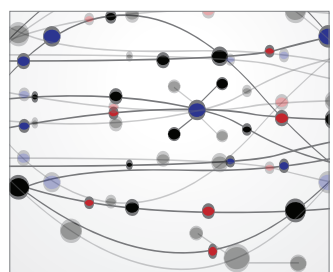

\section{The Scientific} World Journal
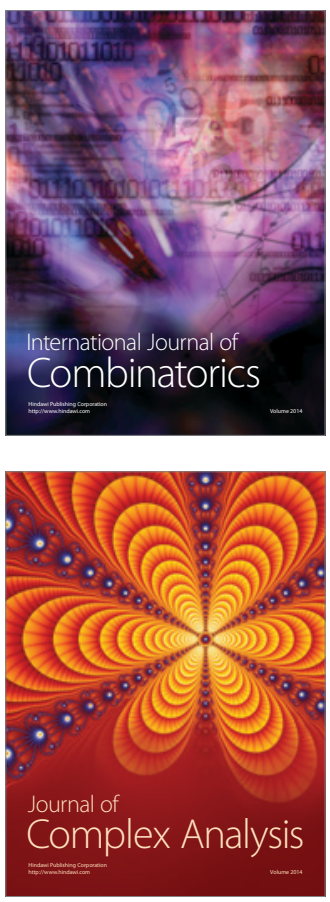

International Journal of

Mathematics and

Mathematical

Sciences
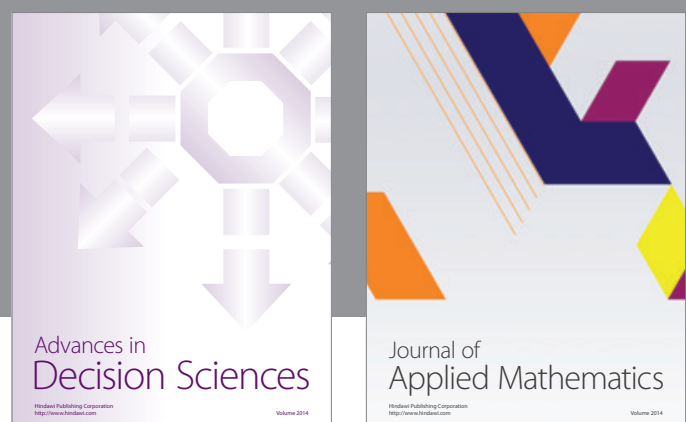

Journal of

Applied Mathematics
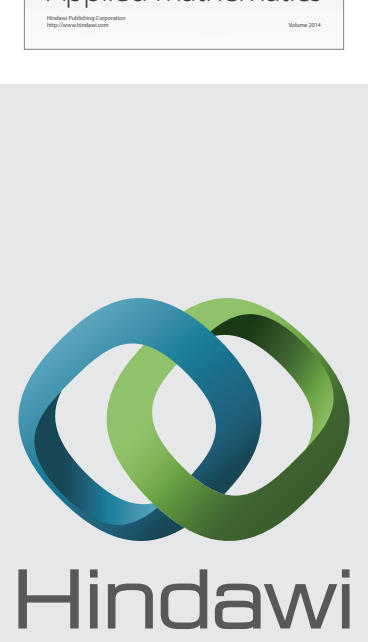

Submit your manuscripts at http://www.hindawi.com
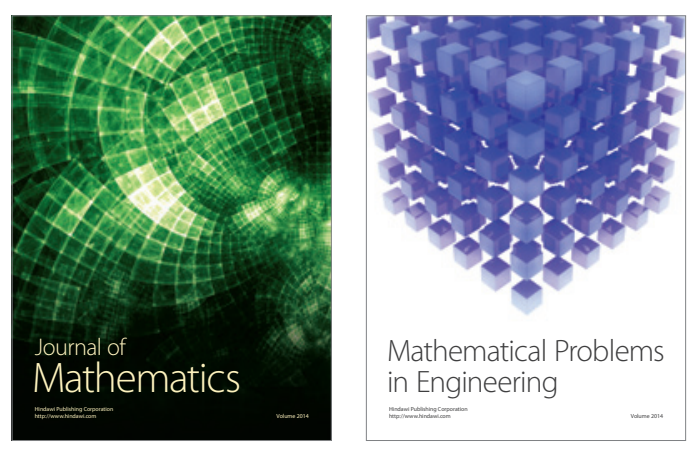

Mathematical Problems in Engineering
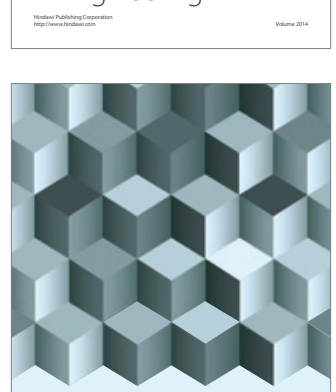

Journal of

Function Spaces
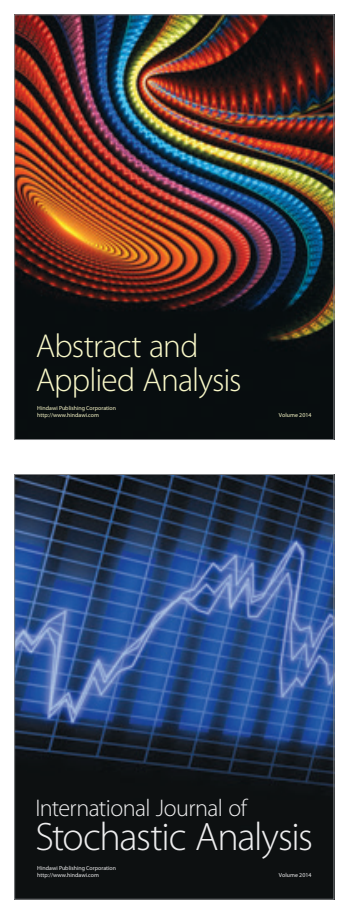

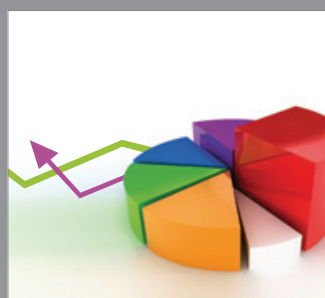

ournal of

Probability and Statistics

Promensencen
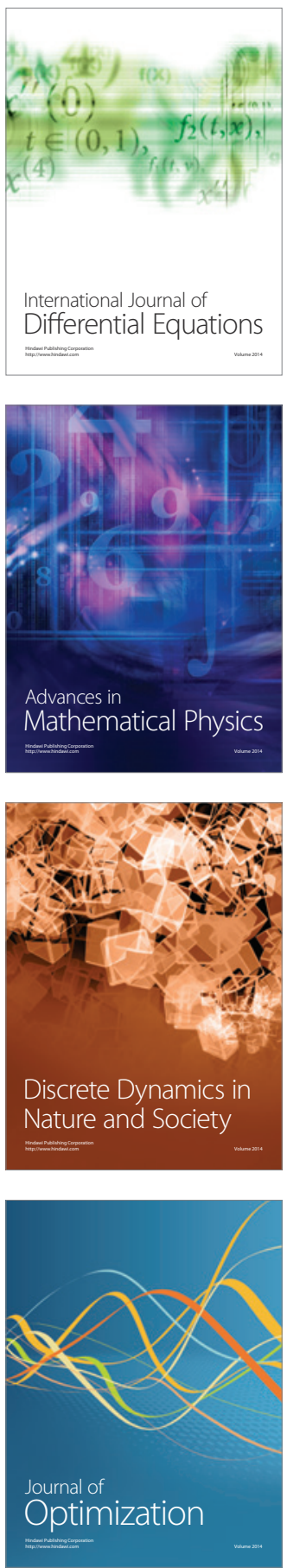